\title{
Measurements investigating the embedding procedure of the Wendelstein 7-X non-planar coils
}

\author{
M. Endler*, T. Bräuer, T. Kluck, J. Müller, L. Wegener \\ Max-Planck-Institut für Plasmaphysik, EURATOM Association, \\ Wendelsteinstraße 1, 17491 Greifswald, Germany
}

\begin{abstract}
One manufacturing step of the Wendelstein 7-X non-planar coils is intended to generate a mechanical prestress between winding pack and casing. To document this "embedding" procedure, we performed several temperature and electric resistivity measurements and geometric surveys before, during and after the embedding. We also investigated the behaviour of quartz sand, used to fill the gap between winding pack and casing, under pressure. The results of the whole body of investigations are consistent with the assumption that the achieved prestress is significantly lower than initially intended.
\end{abstract}

Key words: Wendelstein 7-X (W7-X), Fusion, Stellarator, Superconducting magnet

PACS: 45.70.-n, 52.55.Hc, 81.70.-q, 84.71.Ba, 89.20.Bb

\section{Introduction}

Wendelstein 7-X (W7-X) is a large stellarator under construction at the Greifswald site of Max-Planck-Institut für Plasmaphysik [1,2]. The confining magnetic field will be gererated by a set of 50 non-planar and 20 planar superconducting coils. In each coil, the winding pack (WP) is contained in a steel casing for structural reinforcement. The WP is wound from a cable-in-conduit conductor (CICC) of which the aluminium jacket largely determines the modulus and the thermal expansion coefficient. In the non-planar coils, a compound of

* Corresponding author
Email address: endler@ipp.mpg.de (M. Endler). 
quartz sand and resin fills the gap between WP and casing for a tight connection between the two components. More detailed information about the magnet system and its manufacturing in general can be found in [3].

Even if a tight connection between WP and casing is established by the embedding at room temperature, the WP, from aluminium, would shrink stronger than the steel casing when cooled down to the operating temperature of $4 \mathrm{~K}$, such that gaps might open. Once the current is ramped up and causes the WP to deform due to electromagnetic forces, this could lead to a movement of the WP within the casing. (The readings of strain gauges on the Swiss LCT coil as documented in [4, in particular pp. 121-122/figs. 5.46-5.48, 6.12 and 6.38] were interpreted to reflect such an effect.)

In order to obtain a tight connection between WP and casing at operating temperature, a suitable prestress between the two components should be achieved by a special manufacturing step, the embedding procedure.

After describing this step in some more detail in section 2, we shall present the measurements performed to document this step and to explain deviations from the expectation in sections $3-5$, before drawing conclusions in section 6 .

\section{Embedding procedure}

For the welding of the two half shells of the casing with the WP inside, the coil is mounted on a machine table on which it can be turned and tilted. The gap between WP and casing is still empty. On each side of the WP, four aluminium plates with fitted holes are fixed. The fitted holes serve as reference marks ("pins") for geometrical surveys of the WP shape. To this end, openings in the casing above the pins allow access. The coil is positioned upright. The WP is held in place within the casing by a block at the bottom in vertical direction and by bolts pressing onto the pin plates in horizontal direction (see fig. 1).

Next, sharp quartz sand with rather uniform grain size is filled into the gap between the WP and the casing and distributed by turning and tilting the coil. The casing is then heated to $\sim 120^{\circ} \mathrm{C}$. The sand is compacted by vibrating the coil. It should thus form a tight connection between WP and casing, and, due to its sharp edges, should no longer move once the vibrating is stopped - like the ballast in railway engineering. The casing then cools down back to room temperature and should compress the WP, thus introducing the desired prestress. The temperature of $120^{\circ} \mathrm{C}$ is chosen such that the coil would be free of stress at operating temperature due to the larger thermal expansion coefficient of aluminium relative to steel $\left(1.65 \cdot 10^{-5} \mathrm{~K}^{-1}\right.$ versus $2.3 \cdot 10^{-5} \mathrm{~K}^{-1}$ 


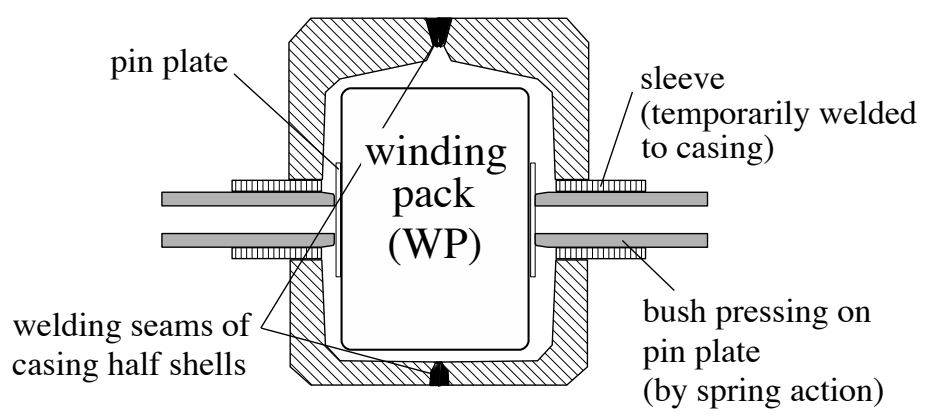

Figure 1. Cross section of a W7-X non-planar coil at the location of a pair of pin plates (schematically). The winding pack (WP) is held in place in horizontal direction by bushes pressing onto the pin plates, before the gap between WP and casing is filled with a sand-resin compound.

at room temperature).

\section{Geometrical surveys before and after the embedding}

Before and after the embedding procedure, four of the eight reference marks (pins) on the WP and corresponding reference marks on the casing were surveyed geometrically with an articulated arm. The 3D measurement uncertainty of these surveys is $\sim 0.3 \mathrm{~mm}$.

For a coil of circular shape with the same cross-sectional areas of WP and casing and same circumference, from the Young's moduli of aluminium and steel, an overall compression of $\sim 10 \mathrm{~mm}$ for the WP and expansion of $\sim 2 \mathrm{~mm}$ for the casing would result along the perimeter of the coil, assuming a temperature difference of $80 \mathrm{~K}$ at the locking between WP and casing. This corresponds to a radial compression of $\sim 1.5 \mathrm{~mm}$ of the $\mathrm{WP}$ and a radial expansion of $\sim 0.3 \mathrm{~mm}$ of the casing. A detailed finite element model taking into account the 3D shape of the non-planar coils and the mechanical properties of the sand-resin compound and of the insulating layers of the WP yields rather similar results $[5,6]$.

The predicted deformation of the WP should be easily detectable within the accuracy of the geometrical surveys, whereas for the casing it would not much exceed the measurement accuracy and therefore require the statistical analysis of surveys from several coils. However, once the surveys before and after the embedding procedures of the first coils were available, no significant compression of the WPs was found. This is demonstrated in fig. 2 for the case of coil AAB13. 


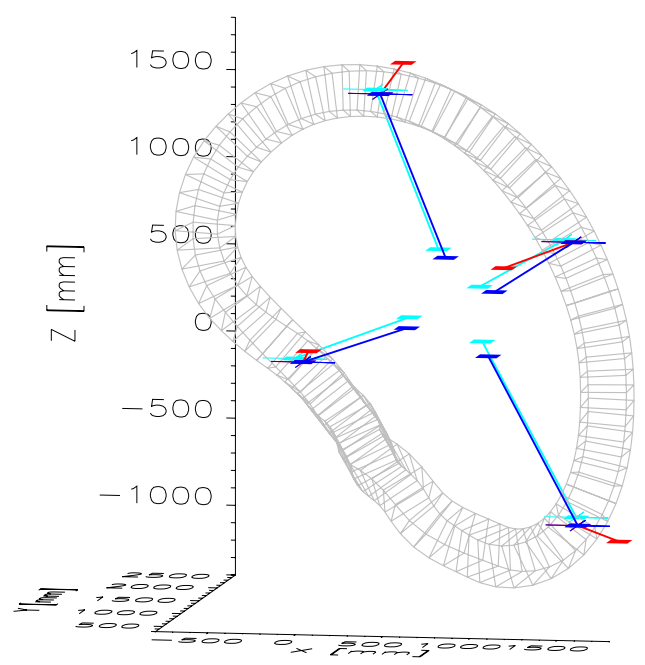

Figure 2. Shifts of the winding pack (WP) reference marks (pins) due to the embedding: pins 1-4 as measured (red) and 1-8 as calculated [6] (light and dark blue). The shifts are multiplied by a factor of 750 for display. Only the calculated shifts (1-1.5 mm) display the expected compression of the WP. The measured shifts are not systematic and of a magnitude not much exceeding the measurement uncertainty.

Various hypotheses were brought forward about the possible reasons for this discrepancy:

(1) The pin plates might be sheared off the WP, since they are at the same time used to hold the WP in place during the embedding (see fig. 1). This had indeed been observed in the very first non-planar coil manufactured, before the connection of the pin plates to the WP was improved. If this hypothesis was true, the pins would no longer represent the WP geometry after the embedding.

(2) A cross beam is screwed to the coil casing to hold the vibration motor in place. The temperature of this cross beam does not increase much while the casing is heated. It might therefore restrict the thermal expansion of the casing.

(3) The expected deformation was calculated under the assumption that the WP stays basically at room temperature while the casing is heated up to $120^{\circ} \mathrm{C}$. This is why care had been taken to achieve a quick heating within $\sim 1 \mathrm{~h}$. If the WP heats up significantly before the vibrating is stopped, a lower prestress would be achieved.

(4) It was observed that the final casing temperature varied by $\sim 30 \mathrm{~K}$ in different locations on the casing. This could lead to a non-uniform thermal expansion and hence to deviations from the expected final deformation of the WP, although one would still expect an overall shrinkage.

(5) The modulus of the compacted sand might deviate from that of the hardened sand-resin compound, which was used for the finite element model predicting the deformations. If the sand layer in the gap between WP and 
casing is compressed significantly once the casing shrinks back to room temperature, the radial compression of the WP would correspondingly be lower.

(6) If, finally, the compacted sand would not stay in place under the pressure of the shrinking casing but rather flow around the WP to the inner side of the coil, the pressure could not be transmitted to the WP, which would not be deformed.

In sections $4-5$ we describe the measurements performed to test these hypotheses.

\section{Multiple measurements during the embedding process}

During the embedding procedure of coil AAB51, the following measurements were performed repeatedly:

(1) surface temperature of the coil casing with a contact thermometer in $\sim 30$ locations and of four pin plates

(2) ohmic resistivity of WP and casing to obtain average temperatures

(3) videoscopic observation of the positions of the bushes relative to the pin plates to verify the expansion of the casing relative to the WP

(4) photogrammetric surveys of the casing to directly verify the thermal expansion of the casing and its uniformity.

The average casing temperature derived from resistivity agreed well with the surface temperatures within the measurement accuracy. The same was true for the average WP temperature and the surface temperatures on the pin plates. A scatter of surface temperatures of typically $20-30 \mathrm{~K}$ and up to $50 \mathrm{~K}$ was observed (standard deviation 10-13 K). During the heating (which is achieved from the outside by heating mats covering the entire coil surface), the surface temperatures were rather larger than the average temperatures. The reverse was true during cooldown of the coil, which appears to be a reasonable result.

The photogrammetric surveys confirmed the expected expansion of the casing (see fig. 3). Anisotropies of the expansion with scales of $0.5-1 \mathrm{~m}$ were observed, which are very probably due to the non-uniform temperature of the casing, since the amount of anisotropy correlates with the standard deviation of the surface temperature distribution even for the same average temperature (the temperature variations were larger during the heating phase than during cooldown).

The cross beam holding the vibration motor was found not to restrict the expansion of the casing (hypothesis (2) of section 3). 


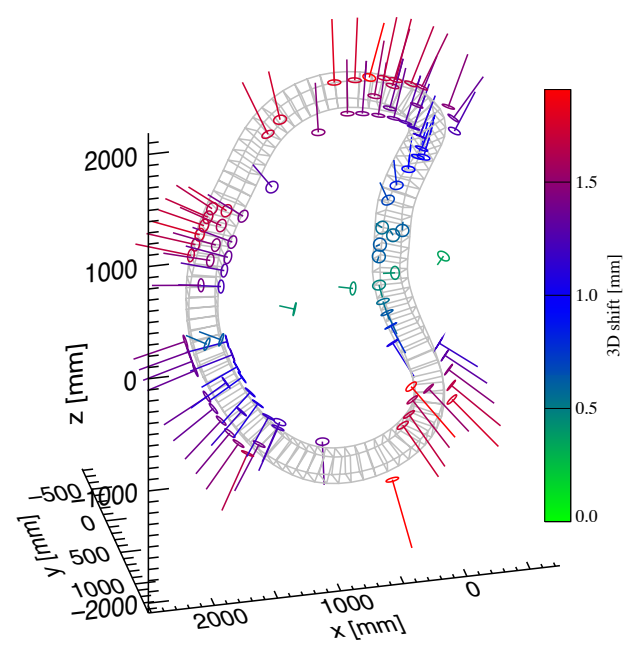

Figure 3. Thermal expansion of the casing of coil AAB51 relative to the cold state, as measured by photogrammetry, at a casing temperature of $80-90^{\circ} \mathrm{C}$, right after the end of the vibrating phase to compact the quartz sand in the gap between winding pack and casing.

The videoscopic observation confirmed movements of the bushes pressing onto the pin plates relative to the pin plates. It is therefore confirmed that the pin plates do not stick to the bushes moving with the expansion of the casing. Rather, the pin plates remain attached to the WP (hypothesis (1) of section 3). This is an important result, since the later geometric surveys rely on these pins as the reference of the WP position.

The pin plates were observed to shift radially inward relative to the bushes until the start of the vibrating phase, since the WP initially expands less than the casing. They then start to move back as the WP temperature increases further during the vibrating phase: at the end of this phase, according to its temperature, the WP should have expanded by almost the same factor as the casing, due to the larger thermal expansion coefficient of aluminium as compared with steel (hypothesis (3) in section 3). This is demonstrated in fig. 4, where we have transformed all temperatures into thermal expansion factors with the corresponding expansion coefficients. We remark that, at the end of the vibrating phase, the expansion of the WP from videoscopy, combined with the photogrammetry of the casing, (blue circles) is slightly smaller than expected from the WP temperature (turquoise squares). This may point to a certain restriction of the further expansion of the WP by the embedding sand. Hence, a certain prestress may have been achieved in the end, which would, however, not exceed $1 / 5$ of the intended value. This is consistent with the observation of no systematic deformation of the WP after the embedding procedure exceeding the measurement uncertainty of $0.3 \mathrm{~mm}$. 


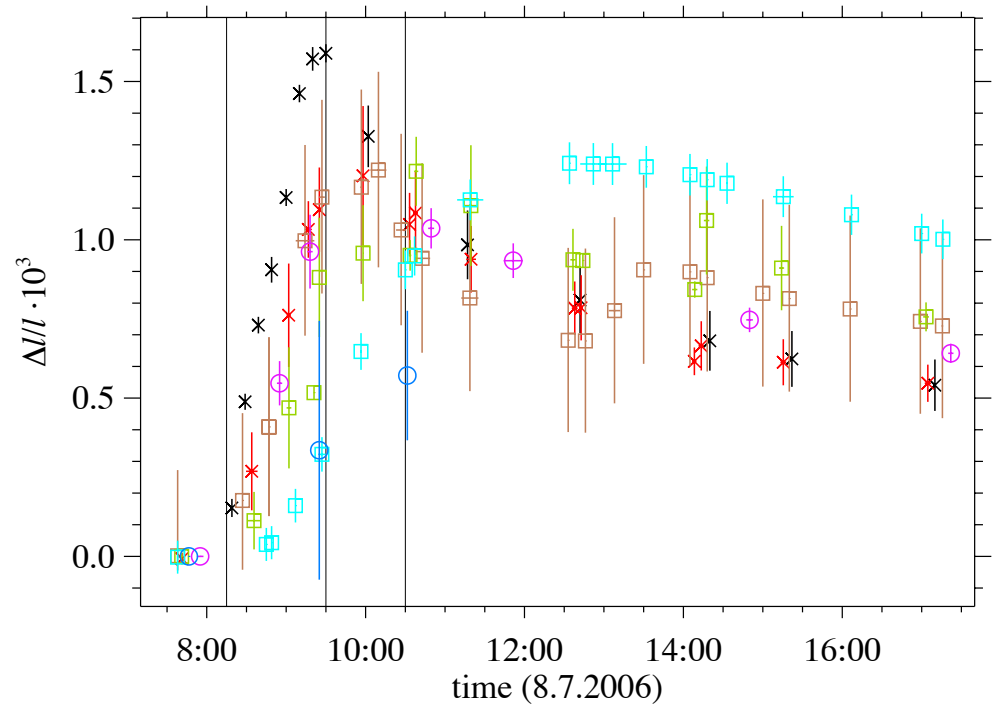

Figure 4. Relative thermal expansion $\Delta l / l$ of winding pack (WP) and casing of coil AAB51 during heating up and initial cooldown of the embedding procedure. Temperatures have been transformed into expansions with the appropriate thermal expansion coefficients. Black crosses: from heating mat control sensors; red crosses: from contact thermometer (casing); green squares: from contact thermometer (pin plates on WP); brown squares: from temperature by resistivity (casing); turquoise squares: from temperature by resistivity (WP); violet circles: from photogrammetry (casing); blue circles: from videoscopy and photogrammetry (WP). The vertical lines indicate (from left to right): start of heating; end of heating and start of vibrating; end of vibrating.

\section{Behaviour of the quartz sand used for embedding}

The compressibility of the quartz sand used to fill the gap between WP and casing was investigated in a compressive hydraulic test machine. To this end, sand was filled into a circular mould and compressed by a piston of $50 \mathrm{~mm}$ diameter. Maximum pressures of $100 \mathrm{MPa}$ could thus be applied. In the detailed finite element model of the embedding procedure, maximum pressures of $\sim 50 \mathrm{MPa}$ and average pressures of $5-10 \mathrm{MPa}$ were predicted for a Young's modulus of $10 \mathrm{GPa}$ of the embedding material. This modulus had been measured for the hardened sand-resin compound.

In our tests, two moulds with holes of $120 \mathrm{~mm}$ depth and of $50 \mathrm{~mm}$ and $80 \mathrm{~mm}$ diameter were used. Before applying pressure to the sand, it was compacted by vertical vibrations of $\pm 0.5 \mathrm{~mm}$ at $16 \mathrm{~Hz}$ to simulate the procedure during the embedding process.

The first mould, tightly fitting to the piston, served to determine the compressibility of the sand. The pressure was increased three times to a maximum of $100 \mathrm{MPa}$ and released to 0 , or, with a new sample of sand, to a maximum 
of $10 \mathrm{MPa}$. The sand did not behave elastically but exhibited a hysteresis in $\Delta s(p)$. Moreover, $\Delta s$ did not reduce to 0 once the pressure was released.

For our purpose, the compressibility in the first compression is the most interesting parameter. Here, we observed values of 0.2-0.4 GPa rather than $10 \mathrm{GPa}$ (see fig. 5 - hypothesis (5) from section 3).

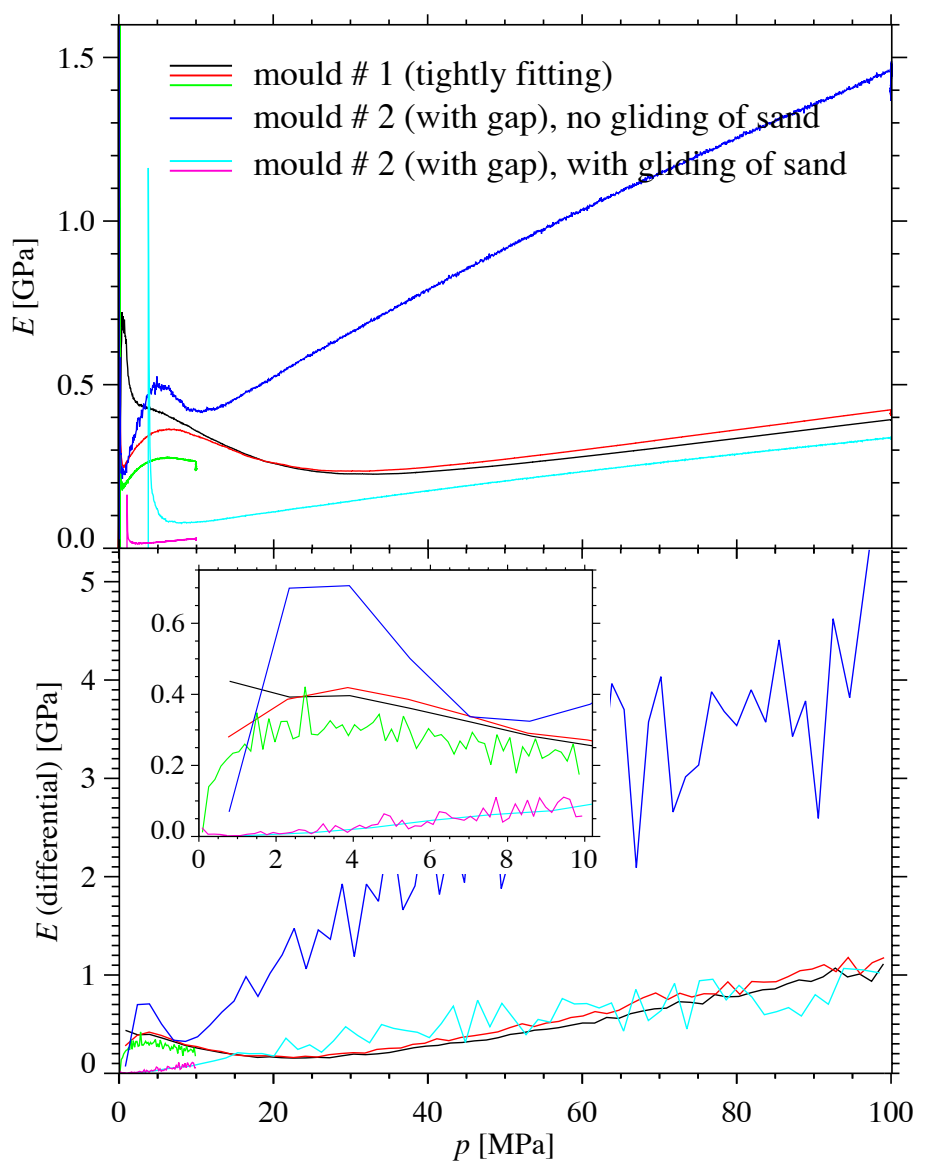

Figure 5. Young's modulus $E$ for the first compression of the quartz sand used for embedding in the $50 \mathrm{~mm}$ mould (\#1) and the $80 \mathrm{~mm}$ mould (\#2). For both moulds, two runs up to a pressure of $100 \mathrm{MPa}$ and one run up to $10 \mathrm{MPa}$ were performed. Top: integral value $E_{\text {int }}=(p / \Delta s) s$; bottom: differential value $E_{\text {diff }}=(\delta p / \delta \Delta s) s$. For the cases with gliding of the sand, $E_{\text {int }}$ is not very reliable, whereas $E_{\text {diff }}$ is calculated from the differences between neighbouring measurement points of $p$ and $\Delta s$. The low-pressure range, most relevant for the understanding of the embedding process, is enlarged in the inset.

With the second mould, a $15 \mathrm{~mm}$ gap remains between mould and piston, which was filled up to $15-20 \mathrm{~mm}$ below the upper plane of the mould. The $15 \mathrm{~mm}$ gap corresponds to the typical width of the gap between WP and casing (see fig. 1). This is to test whether, under pressure, sand will "flow" from below the piston up the gap (hypothesis (6) from section 3). Indeed, in 2 out of 3 runs we observed that sand was pressed from below the piston up into the gap 
between piston and mould, thus reducing the layer of sand below the piston from $\sim 17 \mathrm{~mm}$ to $\sim 5 \mathrm{~mm}$. In both cases, this gliding occured at a pressure below $1 \mathrm{MPa}$, i. e., at a value to be expected everywhere in the embedding. The compressibility of the remaining layer directly after the gliding of the sand up to $\sim 30 \mathrm{MPa}$ was still significantly below that found with the $50 \mathrm{~mm}$ mould, namely as low as $0.02-0.1 \mathrm{GPa}$ up to a pressure of $10 \mathrm{MPa}$ (see fig. 5 , turquoise and violet curves). In contrast, the compressibility up to $10 \mathrm{MPa}$ is of similar magnitude as in the $50 \mathrm{~mm}$ mould if no gliding of sand occurs in the $80 \mathrm{~mm}$ mould (fig. 5, blue curve).

\section{Conclusions}

The photogrammetric surveys of the coil casing show that it expands thermally as expected from its average temperature. Anisotropies of a certain level do exist, presumably due to variations in the casing temperature. However, these anisotropies are too small to affect the general principle of the embedding procedure. Furthermore, no influence was found of the cross beam holding the vibration motor, which was screwed across the coil. Obviously, the screws provide sufficient clearing to decouple the cross beam from the coil, as far as the thermal expansion is concerned.

We observed a movement of the bushes (attached to the casing) relative to the pin plates of the WP in the direction expected during the heating of the casing. We can therefore reject the hypothesis that no deformation of the WP was observed only because the reference marks used were actually sheared off the WP during the embedding procedure and stayed sticking to the bushes. Rather, these pins remain a valid reference of the WP position and shape within the casing. Their use as primary reference marks in later manufacturing stages of the coils is therefore justified.

Tests of the quartz sand filling the gap between WP and casing showed that its compressibility is significantly larger than that of the hardened sand-resin compound. Nevertheless, due to the low thickness of the sand layer, it could still transmit a significant prestress between casing and WP. In addition, however, we observed that the sand layer can glide under a comparatively low pressure, in spite of a preceding compaction by vibrations. Although we took care to simulate the geometrical conditions of the gap between WP and casing in our experiments as closely as possible, such a gliding of the compacted sand around the WP under pressure might still not occur in the real coil geometry.

Finally, the WP was found to heat up before the end of the vibrating phase by such an amount, that its thermal expansion was almost the same as the casing's, due to the higher thermal expansion coefficient of aluminium as com- 
pared with steel. The assumption underlying the embedding procedure, that the WP stays basically at room temperature while the casing is heated to $120^{\circ} \mathrm{C}$, was therefore not fulfilled in the real process. Hence, the results of our measurements during the embedding are consistent with the lack of a systematic deformation of the WP, as derived from geometric surveys before and after the embedding. From the direct videoscopic observation of shifts between casing and pin plates, we conclude that a prestress below $\sim 1 / 5$ of the intended value may still have been achieved. Model computations are planned to investigate the consequences of this result for operating conditions, together with a careful analysis of the readings of strain gauges during the coil tests in their self field.

\section{Acknowledgements}

We thank J. Reißmann and the employees of Babcock Noell for their support in the measurements during the embedding of coils, Mrs. H. Bock of Stralsund University of Applied Sciences and her students for their assistance in determining the properties of the quartz sand, and many colleagues at IPP Greifswald for their help in preparing the measurements and discussing the results.

\section{References}

[1] R. C. Wolf, Wendelstein 7-X Team, Fusion Eng. Des. 83 (2008) 990.

[2] F. Schauer, Fusion Eng. Des. 82 (2007) 443.

[3] L. Wegener, J.-H. Feist, J. Sapper, F. Kerl, F. Werner, Fusion Eng. Des. 58-59 (2001) 225 .

[4] P. N. Haubenreich, P. Komarek, S. Shimamoto, G. Vécsey, L. Dresner, W. A. Fietz, et al., Fusion Eng. Des. 7 (1988) 1.

[5] E. Bondarchuk, A. Boykov, N. Doinikov, V. Komarov, Y. Krasikov, A. Labusov, et al., Analysis of the stress-strain state of the first and third non-planar coils after cooldown to $4 \mathrm{k}$ in the self-field (report no. 1), Tech. rep., Efremov Scientific Research Institute, St. Petersburg (2003).

[6] E. Bondarchuk, V. Komarov, A. Labusov, I. Labusov, A. Panin, Analysis of the stress-strain state of the first and third non-planar coils after cooldown to $4 \mathrm{k}$ in the self-field. First amendment (report no. 2), Tech. rep., Efremov Scientific Research Institute, St. Petersburg (2003). 